\title{
Prendimiento de dos tipos de injertos en cacao en distintas fases lunares, Siuna, 2014
}

\author{
Mario Reyes Martínez ${ }^{1}$ \\ Lesther Marín Mendieta ${ }^{2}$ \\ Oscar Montalván Castellón ${ }^{3}$
}

\section{Resumen}

Sin duda, los productores saben de la influencia lunar y sobre el movimiento de la savia en las plantas en determinados momentos, debido a los conocimientos ancestrales y experiencias adquiridas. Sin embargo, en relación al prendimiento de injertos en cacao (Theobroma cacao), existen pocos estudios científicos que demuestren tales efectos. El objetivo principal del estudio fue comparar los efectos de las fases lunares en el prendimiento de dos tipos de injertos en cacao. Para ello se utilizó un diseño completamente al azar con arreglo bifactorial, con tres repeticiones por cada una de las fases y un universo de 576 plantas. La variable de medición únicamente fue el prendimiento de las plantas que se injertaron.

Los datos fueron procesados en el programa Statistical Product and Service Solutions (SPSS) versión 21 y se sometieron al análisis de varianza ANDEVA con un nivel de confianza del 95\%. Los análisis revelan que existen diferencias significativas para las técnicas de injertación $(\mathrm{p}=0.011<0.05)$ y se obtuvo mayores prendimientos en los injertos de yema sobre los de bisel. Con respecto al efecto de las fases lunares sobre los dos tipos de injertos no se encontró evidencia estadística ( $p=0.950>0.05$ ). En base al análisis combinado de varianza no existen diferencias significativas para la interacción fases lunares y técnicas de injertación puesto que ( $\mathrm{p}=0.761>0.05)$.

Palabras clave: influencia lunar; injertación de cacao; tipos de injertos.

\section{Summary}

There is no doubt the producers know of the moon influence and the sap movement in plants at certain moments, due to the ancestral knowledge and experiences gained. However, in relation to the root taking of grafts in cocoa (Theobroma cacao), there are few scientific studies that demonstrate such effects. The main objective of this study was to compare the effects of moon phases in the root taking of two types of grafts in cocoa. To do this we used a complete randomized design with bifactorial order, with

\footnotetext{
Ingeniero Agroforestal. Docente de URACCAN Las Minas. reyesmario128@yahoo.com

Ingeniero. Agroforestal. Docente de URACCAN Las Minas lesthermarin@yahoo.com

MSc. Docencia universitaria. Ingeniero Agroforestal. Docente de URACCAN Las Minas. montesiuna@yahoo.com
} 
three replays for each of the phases and a universe of 576 plants. The measurement variable was based on the root taking of the plants that were implanted.

The data were processed in the Statistical Product and Service Solutions (SPSS) program (version 21), and subjected to the ANOVA analysis of variance with a reliability level of $95 \%$. The analysis shows significant differences for grafting techniques $(\mathrm{p}=0.011<0.05)$ and higher rooting of the bud was obtained above the bevel's. Regarding the effect of moon phases on the two types of grafts, no statistical evidence was found $(p=0.950>0.05)$. Based on the combined analysis of variance there is no significant differences for the interaction between moon phases and grafting techniques since $(\mathrm{p}=0.761>0.05)$.

Keywords: Moon influence; cocoa graft; types of grafts.

\section{Introducción}

A nivel de países cacaocultores como Ecuador, han investigado la influencia de la luna sobre la multiplicación vegetativa por injertación, resultando de que la luna influye sobre el porcentaje de rendimiento. En Nicaragua, aún no se registran estudios que determinen el efecto de las fases lunares sobre el prendimiento en relación a cacao, debido a que se experimenta con estas nuevas formas de propagación asexual.

Los agricultores tienen la creencia de que la luna influye en las diferentes labores agrícolas desde la preparación del suelo hasta la recolección de frutos. Muchos estudios consideran la luminosidad lunar esencial para la vida y desarrollo de las plantas. Independientemente de creer o no creer en la influencia lunar sobre las plantas, está demostrado que la intensidad de la fotosíntesis es superior a partir de creciente hacia llena y que el mayor incremento de la fotosíntesis en los cultivos se registra en el período intensivo de aguas arriba, el cual está comprendido tres días después de creciente hasta tres días posterior a plenilunio. (Restrepo 2005).

Las fases lunares tienen una relación muy estrecha con el movimiento de líquidos y siendo la savia una sustancia liquida en la que circulan una serie de compuestos especialmente hormonales que estimulan la brotación de yemas y el crecimiento de ramas, se entiende la importancia de conocer en qué fase lunar es más conveniente injertar. (Duran, 2009).

Debido a la falta de información científica con base a este tema, se realizó el estudio para determinar el grado de influencia que ejerce la luna sobre el prendimiento en las dos técnicas de injertación en cacao en las condiciones edafoclimáticas del municipio de Siuna. 


\section{Revisión de literatura}

El injerto: Es una práctica de multiplicación que consiste en unir porciones distintas en la planta, de tal manera que haya soldadura y pase la savia, con la formación de una especie de simbiosis, que constituye un único individuo capaz de crecer y desarrollarse (Muñoz, 2013, p.8).

\section{Principales factores que influyen en el prendimiento del injerto}

Temperatura: Tiene efecto en la formación del tejido del callo. La consolidación del injerto requiere una temperatura que oscila entre los 15-30 grados Celsius siendo la óptima entre 22 y 25 grados Celsius (Corral, 2012, p.43).

Sombra: Una vez hechos los injertos se colocan bajo media sombra (malla Sarán 50\%) para darles el cuidado necesario. Se debe proporcionar sombra al área de propagación para reducir la intensidad lumínica y las altas temperaturas (malla sarán 50 a 70\%; Palma, 2009, p.23).

Humedad: es importante cuando se está formando el callo para que no se deseque la superficie de los cortes realizados, y la cicatrización sea buena. Esta debe estar entre 80 y $90 \%$ siempre elevada, pues en caso contrario la buena cicatrización son reducidas (Corral, 2012, p.43).

Viento: puede tener influencia sobre la humedad y deshidratación de las púas. La gran velocidad del viento puede acelerar la deshidratación de las púas, también puede disminuir el prendimiento, al romper brotes y desprender la unión de los cambium. El promedio de la velocidad del viento debe ser de 4 a 6 metro por segundos $(\mathrm{m} / \mathrm{s})$ (14.4 a 21.6 kilómetros por hora) con ráfagas de 6 a 8 metro por segundos $(\mathrm{m} / \mathrm{s})(21.6$ a 28.8 kilómetros por hora) (km/h), (Muse, 2012, p.23).

Oxígeno: Para la producción del tejido del callo es necesaria la presencia de oxígeno en la zona de unión, debido a que en esta hay un gran número de células en división y crecimiento que lo acompaña una respiración elevada. Para esto es conveniente que la ligadura del injerto permita el acceso del oxígeno a la zona de la unión. (Corral, 2012, p.43).

Compatibilidad: Como regla general, los vegetales injertados deben ser de la misma familia botánica y la afinidad, es mejor entre los vegetales de la misma especie que entre dos vegetales de un mismo género. Normalmente debe haber éxito si se injerta un clon dentro de la misma planta de la cual provino el patrón, injertando en otra planta del mismo clon o clones de la misma especie. (CORPOICA, 2004, p.20). 
Técnicas de injerto: Si se pone en contacto sólo una reducida porción de las regiones cambiales del patrón y de la variedad, la unión será deficiente. Aunque haya una buena cicatrización y comience el crecimiento de la variedad, cuando éste alcance un desarrollo importante, una unión tan escasa impedirá el movimiento suficiente del agua y se producirá el colapso de la planta injertada. La elección de la correcta técnica de injerto es muy importante a la hora de aumentar el éxito en la unión (Corral, 2012, p.43).

Edad del patrón: El patrón estará listo para injertarse entre los cuatro a seis meses de edad, cuando el diámetro del tallo de la planta en vivero alcance un centímetro. De acuerdo a Sian en la evaluación del prendimiento de injerto de cacao (Theobroma cacao L) United Fruit (UF-667), en cinco etapas de crecimiento del patrón Pound-7; el mayor prendimiento se obtuvo a los 120 días de edad del patrón con el 91.25\%, utilizando únicamente el injerto tipo U invertida o de yema (Sian, 2005, p.37).

Contaminación con patógenos: En ocasiones entran en las heridas producidas al injertar, bacterias y hongos que causan la pérdida del injerto. Para prevenir estas infecciones, utilizar agua limpia y manos limpias (Corral, 2012, p.43).

Épocas de injertación: Las épocas favorables para injertar se condicionan a la clase de plantas, estado vegetativo, así como las condiciones edafoclimáticas del lugar. Dependiendo de la época, clase de injerto que se adopte y de las precauciones que se pueden tomar; los meses de febrero a mayo generalmente son las mejores épocas para realizar la labor de injerto (Paredes, 2010, p. 31).

Tiempo de injertación: En el caso del cacao (Theobroma cacao), el tiempo máximo que debe transcurrir al realizar los procedimientos de injertación por individuo no debe exceder de 30 segundos en promedio (Paredes, 2010, p.35).

Hora de injertación: Según Santana en su estudio para determinar el tipo de injerto y la hora efectiva de realización sobre el prendimiento de yemas en plántulas de cacao, determinó que, la realización de la labor de injertación en horas de la mañana (o8:00 a. m), favorece el prendimiento de las yemas en los patrones. (Santana, 2013, p.46).

Grosor del tallo: El grosor del tallo presente en el patrón también influye sobre el prendimiento de las yemas, debido a que a mayor diámetro del patrón mayor cantidad de tejido vegetal para que la yema pueda acoplarse a este. Es preferible que el patrón y la púa tengan el mismo diámetro. (Santana, 2013, p.43).

Cuidados después de la injertación en vivero: Una vez injertadas las plantas deben protegerse del sol y tener cuidado al aplicar el riego, porque debe ser controlado para evitar entradas de agua en la unión del injerto, asimismo después de injertar se elimina la yema apical del patrón de esta forma se estimulara la brotación del injerto. (Orantes, 2003, p.33). 


\section{Las fases de la luna}

a) De luna nueva a creciente: Se conoce como la fase de la luna nueva, período que en el subsuelo se producen, entre otras cosas, grandes movimientos de agua que afectan directamente las actividades agrícolas, la disponibilidad de luz lunar va en aumento y las plantas tienen un crecimiento balanceado, en el que se favorece el crecimiento de follaje y raíz. (Calderón, 2012, p.6).

b) De cuarto creciente a luna llena: Esta fase se conoce como cuarto creciente. En este período sigue aumentando la luz lunar y hay poco crecimiento de raíces, pero mucho crecimiento del follaje. Aquí se incrementa cada día la luz reflejada aumentando la fuerza vital o el vigor de las plantas. Las plantas cuentan con una mayor cantidad y movimiento interno de agua. La radiación favorece la cicatrización de los tejidos y órganos, lo que ayuda a eliminar las células muertas. (Duran, 2009, p.17).

c) De luna llena a cuarto menguante (la fase luna llena): En este período, la dinámica de los fluidos llega a su punto máximo, especialmente cerca al cuarto día de esta fase, por lo que se fomenta el poder germinativo y el crecimiento; la savia bruta asciende con gran poder nutritivo, por lo que no es recomendable realizar labores que afecten en forma extrema a los órganos de las plantas y se trata entonces de un período de crecimiento conservador sobre todo en los últimos días de esta fase. (Calderón, 2012, p.8).

d) De cuarto menguante a luna nueva (fase menguante): Durante esta fase los fluidos disminuyen su dinámica y se presenta el punto más bajo, cerca del quinto día de esta fase; ahora la savia ha empezado a descender, potencializando aquellas actividades que involucran forma, calidad alimenticia y resistencia, por lo que es una fase principalmente de conservación, en donde las plantas se fortifican. (Olivo, 2013, p.32).

\section{Influencia de las fases lunares en el movimiento de la savia de las plantas}

Se ha comprobado que los cambios lunares se hacen sentir en la savia de las plantas, iniciándose el proceso de su influencia desde la parte más elevada para ir descendiendo gradualmente a lo largo de todo el tallo, hasta llegar al sistema radical. (Restrepo, 2005, p.4).

La luz lunar coadyuva a la cicatrización de vegetales que han sido amputados y desgajados. La luz solar a veces es tan intensa que llega a interrumpir este proceso o puede dañar las amputaciones expuestas; se propicia la interacción nutritiva de las plantas y resulta benéfico porque los nutrientes fluyen más rápido, en las cortezas de los injertos y se acelera el proceso de formación de callo. La luz lunar acelera el crecimiento de muchos vegetales, entre ellos las yemas de las púas injertadas. El rápido crecimiento de las yemas es fundamental en este arte de los injertos. (Montes, 2010, p.32). 


\section{Prendimiento de injertos en las diferentes fases lunares}

En el año 2008 en Ecuador, Yance Molina, en su estudio de diferentes sistemas de injerto examinado al efecto de las fases lunares sobre la multiplicacion asexual de (Theobroma cacao), analizó los promedios porcentuales de prendimiento de los diferentes clones en los métodos de injertación sometidos a la influencia de las cuatro fases lunares alcanzando una alta significancia estadística,esto según el dendrograma del metodo de Ward's.

Para un mejor reflejo de la variable se resolvió como guía el indicador que el mayor promedio de $96 \%$ se tiene a partir de la fase creciente, al clon Colección Castro Naranjal (CCN-51) con el método de ramilla terminal (bisel), seguida con un 88\% para el mismo clon, pero en fase lunar llena con el tipo de Púa Lateral (PL). Los promedios de entre 50 y más de 50\% están en luna llena y menguante para los clones Estación Experimental Tropical (EET-48, 96 y 103) para cualquiera de los métodos usados. Mientras que los promedios menores u oscilantes a 50\% mayormente fueron dados a partir de luna nueva con la excepción de que un 80\% lo alcanzó el clon Estación Experimental Tropical (EET-48) bajo el tipo de yema y un 70\% bajo el tipo de Púa en la misma fase, fue el clon EET-103 siendo los 2 únicos valores más altos de esta fase, así llegó aumentando a 82\% el clon Colección Castro Naranjal (CCN-51) con el tipo Púa Lateral en la fase creciente.

\section{Efectos de las fases lunares en injertos}

Algunos estudios recomiendan que las labores de injertación, se realicen durante el período de luna llena, esto se debe básicamente a que los cortes hechos en luna llena conservan la madera, por tanto frena el desarrollo de las yemas, de esta manera favorece la unión del injerto. (Márquez, 2001, p.6).

En cuanto a los injertos y las podas, dado que tanto unos como otros representan un traumatismo o una herida en las plantas, las opiniones son diferentes, porque mientras unos creen en la convivencia de realizarlos en la fase de la luna menguante para evitar al máximo la pérdida de savia, otros consideran que los efectos purificadores del plenilunio (luna llena) evitan infecciones y favorecen la cicatrización de heridas. (Rojas, 2010, p.33).

Los injertos de púa y escudo se realizarán en creciente, por el motivo y razón de que nos interesa un desarrollo vegetativo máximo sin encontrarnos ante el problema de que aparezca algún fruto, que merma considerablemente el buen fin del injerto. Por otra parte, no olvidemos que la luna vieja (llena, menguante) posee la virtud de conservar la madera (Angles, 1996, p.16). 


\section{Interacción luna-injerto}

En su investigación Molina (2008), estudió los diferentes sistemas de injerto, examinando al efecto de las fases lunares sobre la multiplicación asexual de cacao (Theobroma cacao L.), también determinó la correlación e interacción de los tratamientos basados en el análisis de las fases lunares frente a los tipos de injertos practicados en los clones, originando así características que van de un o,385\% de relación para pocos casos dentro de lo que puede influenciar la luna para los tres tipos de injertos practicados según dendrograma del método de Ward's.

El dendrograma, señala las similitudes de los parámetros indicados, forma dos grandes grupos en el que el primero de abajo hacia arriba revela un subgrupo formado por la influencia que tiene la fase lunar creciente y llena para los injertos de Yema y Púa los más igualados. El mismo subgrupo es abarcado por la influencia que tiene las fases luna nueva y creciente, para nuevamente los injertos Púa y Yema. Mientras que hay suficiente igualdad en la influencia de la luna nueva en el tipo Yema y Púa Terminal. El segundo grupo muestra que a partir de la fase creciente empieza a mantener un equilibrio los métodos de Ramilla Terminal y se mantienen así en luna llena hasta la menguante el método de Púa Lateral. En la fase menguante se aprecia una distinta influencia para el método de yema formándose así el último subgrupo del dendrograma.

En la respuesta de datos registrados en comparación con tan solo las fases lunares, se identifica solo a las cuatro fases lunares como datos evaluados y principalmente usado en los tratamientos, originando así características que van de un $0,75 \%$ de relación o similitud para las fases llena y menguante y un 1,2\% de desigualdad apartando a la fase lunar creciente y aún mayor alejada con un $2 \%$ la luna nueva dentro de lo que puede influenciar las fases lunares para los tres tipos de injertos practicados, según dendrograma del método de Ward`s.

Lo que detalla es una similitud bastante apegada entre las fases lunares llena y menguante probadas con los otros factores en estudio y quedando así por los resultados de las variables que se estudiaron, impulsando con una distancia no tan lejana a estas dos fases la luna creciente, separada por factores que el estudio presentó.

\section{Materiales y métodos}

\section{Ubicación del experimento}

El estudio se realizó en el Laboratorio Natural de la URACCAN, comunidad del Hormiguero municipio de Siuna, presenta clima tropical húmedo, suelo entre franco arenoso a franco arcilloso, la pendiente entre un rango de 2 a 10\% con vientos que van del suroeste a noroeste, pluviosidad de 1500 a 2500 mm/año y 114 metros sobre 
el nivel del mar (msnm), con coordenadas Universal Transverse Mercator (UTM) X: 708364; Y: 1518634.

Se tomaron mediciones de los datos ambientales durante el período de ejecución de la investigación a través de la estación meteorológica del INETER (Instituto Nicaragüense de Estudios Territoriales) ubicados en el Laboratorio Natural de la universidad URACCAN, esto con el fin de obtener resultados más precisos en cuanto a la influencia lunar sobre los injertos y desestimar que fueron factores climáticos los que intervinieron en los resultados. Durante el período de ejecución comprendido del 7 de febrero al 23 de mayo del 2014, se registraron en promedio por mes temperaturas de 26 grados Celsius, humedad relativa de $77 \%$, velocidad del viento o. 55 metros por segundo, precipitación 3.6 milímetros y radiación solar $18 \mathrm{MJ} / \mathrm{m}^{2}$; agregado a esto a que el vivero estaba protegido por malla sarán al 50\% y los injerto se regaban manualmente con regadera.

\section{Diseño experimental}

Se utilizó un diseño bifactorial completamente al azar (BDCA), con 8 tratamientos, 3 repeticiones y 24 unidades experimentales de tamaño de $0.54 \mathrm{~m}^{2}$ cada una, con una población de (576) plantas; teniendo en cuenta (24) plantas evaluadas por unidad experimental.

\section{Material genético}

El material genético para los patrones de cacao y las varetas con las cuales se realizaron los injertos, se obtuvieron del jardín clonal del Laboratorio Natural URACCAN - El Hormiguero, estos clones fueron los, Siuna Guayabo (SAGU-53, SAGU-59) y Siuna Carao (SACA-36, SACA-27).

\section{Manejo agronómico}

En el mes de septiembre del 2013 se procedió a visitar el laboratorio natural "Los laureles” de la URACCAN con la finalidad de seleccionar y limpiar el área de estudio, para ello se utilizaron machete, azadón, piochas, limas. El área seleccionada para el vivero tenía dimensiones de 13.80 × 8 metros, estas mediciones se realizaron con una cinta métrica de $5 \mathrm{~m}$ de longitud.

Una vez preparada el área, se continuó con la preparación del material o sustrato con los cuales se llenaron bolsas de dimensiones de 10 x 8 pulgadas. Con la utilización de piochas, palas, sacos y saranda se procedió a extraer y preparar la tierra con la cual se llenarían 650 bolsas de polietileno negro. Se realizó un semillero donde se colocaron las semillas de cacao para que germinaran y crecieran hasta un determinado tiempo 
no mayor de quince días, con la finalidad de seleccionar las plántulas de mejor vigor y de esa manera procurar obtener patrones sanos.

Para realizar los injertos se tomaron patrones que cumplían los requisitos técnicos establecidos como diámetros de $1 \mathrm{~cm}$, libre de plagas, enfermedades y bifurcaciones o daños mecánicos y a criterio personal solo se injertaron patrones de 4 meses de edad. En cada período que se realizaban los injertos se recolectaron las varetas muy de mañana 7:00 a.m, se continuó a realizar injertos de bisel primeramente para evitar la deshidratación porque a este se le hacen mayores incisiones que al de yema.

Para las actividades de injertación se utilizaron tijeras de podar, navajas de injertar, cintas plásticas transparentes finas para el amarre de la zona injertada, bolsas plásticas transparentes de 5 libras para la protección de los injertos de bisel, desinfectante, cintas plásticas para la identificación en la cual se escribía la fecha de injertación, clon utilizado y el realizador del injerto, agregado a esto que se injertaba el propio día de la fecha lunar para ello se utilizó un calendario lunar para ver los días de cambio.

Variables evaluadas: La única variable evaluada fue el prendimiento de los injertos en las distintas fases lunares.

Análisis estadístico: Para evaluar las diferencias entre tratamientos según la variable a medir, los datos fueron sometidos a un análisis de varianza (ANDEVA), con un nivel de confianza del 95\%, para ello se utilizó el programa estadístico SPSS v21.

\section{Resultados y discusión}

Cuadro No. 1. Prendimiento en los dos tipos de injertos en las diferentes fases lunares

\begin{tabular}{|l|l|r|r|l|}
\hline \multicolumn{5}{|c|}{ Estadísticos descriptivos } \\
\hline \multicolumn{7}{|c|}{ Variable dependiente: Prendimiento (\%) } \\
\hline Técnicas de injerto & \multicolumn{1}{|c|}{ Fases lunares } & \multicolumn{1}{c|}{ Media } & Desviación típica & \multicolumn{1}{c|}{ N } \\
\hline & Luna Llena & 91.6667 & 4.16500 & 3 \\
\hline & Luna Menguante & 93.0567 & 2.40178 & 3 \\
\hline Bisel & Luna Nueva & 91.6667 & 7.21688 & 3 \\
\hline & Luna Creciente & 87.5000 & 15.02544 & 3 \\
\hline & Total & 90.9725 & 7.71081 & 12 \\
\hline & Luna Llena & 97.2233 & 4.80933 & 3 \\
\hline & Luna Menguante & 98.6100 & 2.40755 & 3 \\
\hline & Luna Nueva & 98.6100 & 2.40755 & 3 \\
\hline & Luna Creciente & 100.0000 & .00000 & 3 \\
\hline & Total & 98.6108 & 2.71376 & 12 \\
\hline
\end{tabular}


Se encontraron diferencias estadísticas entre las técnicas de injertación tipo bisel $y$ de yema en base al porcentaje de prendimiento $(p=0.011)$, lo que indica que al menos uno de los tratamientos tiene un promedio diferente, siendo evidente que el injerto de yema obtuvo mejores porcentajes de prendimiento.

La razón por la cual el prendimiento se ve más favorecido en luna menguante utilizando la técnica de injertación de bisel, se debe a que en este período la savia está en descenso y la mayor afluencia de ésta se concentra en el tallo, ya que los cortes realizados en el patrón durante esta fase favorecen una rápida cicatrización del mismo y por ende benefician el prendimiento de los injertos, esto lo manifiesta Molina Borbotó (2014). Estos resultados difieren a los encontrado por Molina en su estudio de diferentes sistemas de injertos examinado al efecto de las fases lunares sobre la multiplicación asexual de cacao donde el mayor prendimiento del injerto de ramilla terminal (Bisel) se obtuvo en la fase lunar creciente con un $96 \%$, seguido por un $74 \%$ en luna menguante de acuerdo a los clones utilizados.

La luna creciente, favorece un mejor prendimiento para la técnica de injertación tipo yema, ya que en ésta etapa la savia va en ascenso, concentrándose principalmente en tallo del patrón, agregado a esto a que los cortes realizados en el mismo son menores en comparación a otras técnicas de injertación, estimulando una rápida cicatrización de dichos cortes, igualmente lo manifiesta Molina Borbotó (2014). Estas medias porcentuales igualmente divergen con los resultados de Molina, ya que en su análisis indica que la técnica tipo yema obtuvo el mayor prendimiento en luna nueva (80\%), continuamente en luna llena con un $76 \%$ para los clones en estudio.

Molina (2008), indica que dentro de los diferentes tipos de injertos que se pueden practicar en cacao, el que mejor resultados ha dado es el de yema, ya sea de escudete o parche. El método de injertación que mayor porcentaje de prendimiento presenta es el método de "parche" el cual registra un porcentaje muy alto respecto a otros métodos utilizados (lateral y aproximación), manteniéndose en un rango del $88 \%$ al $100 \%$ de prendimiento. (Cantero, 2012, p.41).

El trabajoso injerto de parche no es el único o más acertado método de propagar cacao asexualmente, porque actualmente se cuenta con el tipo escudete, púa doble o simple (lateral) de los cuales sus resultados han variado en distintos países. Así por ejemplo, mientras en Ecuador se han obtenido resultados bastante buenos, los que se han obtenido en Costa Rica no son satisfactorios. (Molina, 2008, p.19). 
Cuadro No. 2. Efectos de las fases lunares en los dos tipos de injertos

\begin{tabular}{|l|l|r|r|r|}
\hline \multicolumn{5}{|c|}{ Vases lunares } \\
\hline \multirow{2}{*}{ Fases lunares } & \multicolumn{1}{|c|}{ Media } & \multicolumn{1}{|c|}{ Error típ. } & \multicolumn{2}{c|}{ Intervalo de confianza 95\% } \\
\cline { 4 - 5 } & & & Límite inferior & Límite superior \\
\hline Luna Llena & 94.445 & 2.645 & 88.839 & 100.051 \\
\hline Luna Menguante & 95.833 & 2.645 & 90.227 & 101.439 \\
\hline Luna Nueva & 95.138 & 2.645 & 89.532 & 100.744 \\
\hline Luna Creciente & 93.750 & 2.645 & 88.144 & 99.356 \\
\hline
\end{tabular}

Con respecto a los efectos de las fases lunares en los dos tipos de injertos no se encontró evidencia estadística que demuestre que existan diferencias significativas entre los tratamientos fases lunares puesto que $(\mathrm{p}=0.950>0,05)$. Es en la fase de luna menguante en la cual es más evidente que se logran mejores estimaciones de prendimiento para ambas técnicas de injertación agrupadas, pues algunos autores consideran que es más conveniente realizar los injertos en la fase de luna menguante para evitar la pérdida de savia. (Duran, 2009, p.18).

Estos valores no coinciden a los obtenidos por Borbotó en su estudio, influencia de las fase lunares sobre la reproducción vegetativa de ramillas de diferentes variedades de cacao, indicando que la siembra de ramillas bajo condiciones de luna llena (91.5\%), y luna menguante $(86.5 \%)$ estimulan porcentajes de prendimiento mucho más consistentes en relación con las otras fases lunares utilizadas en el proceso probablemente por el efecto sobre el tejido, lo cual concuerda con Argüello (2000), quienes en investigaciones realizadas ha comprobado que los fenómenos lunares se hacen sentir en la savia de las plantas, iniciándose el proceso de su influencia desde la parte más elevada para ir descendiendo gradualmente a lo largo de todo el tallo, hasta llegar al sistema radical. (Borbotó, 2014, p.32).

\section{Interacción entre los dos tipos de injertos y las fases lunares}

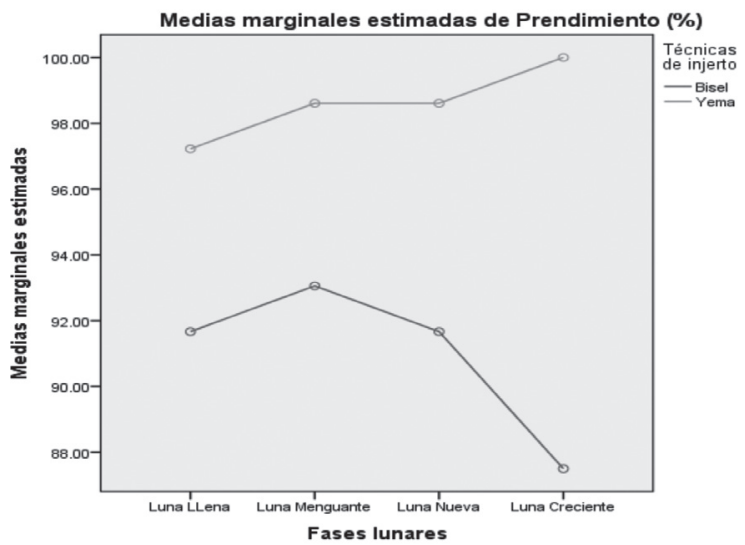

Figura No. 1. Efecto de interacción entre los dos tipos de injertos y las fases lunares 
En relación a la interacción fases lunares y técnicas de injertacion no se encontró evidencia estadística $(\mathrm{p}=0.761>0.05)$.

La fase luna creciente propicia un mayor prendimiento en la técnica de injertacion tipo yema, ya que es en ésta etapa cuando la savia está ascendiendo, concentrándose principalmente en el tallo del patrón, agregado a esto a que los cortes realizados en el mismo son menores en comparación a otras técnicas de injertación, estimulando una rápida cicatrización de dichos cortes, igualmente lo manifiesta Molina Borbotó (2014).

Estos resultados igualmente discrepan a lo encontrado por Molina, quien evaluó el prendimiento de tres métodos de injertación en las diferentes fases lunares en cacao, estableciendo que es la fase luna nueva en la cual la técnica tipo yema alcanza el mayor porcentaje de prendimiento con un $80 \%$.

\section{Conclusiones}

Acorde al porcentaje de prendimiento de las dos técnicas de injertación en las distintas fases lunares el análisis estadístico reveló que existen diferencias significativas entre ellas, $(\mathrm{p}=0.011<0.05)$. se establece de que las mayores medias porcentuales se obtienen en la técnica de injertación tipo yema en la fase lunar creciente $(100 \%)$, mientras que para la técnica tipo bisel en menguante (93.0567\%).

De acuerdo a los efectos de las fases lunares en los dos tipos de injertos, no se encontró evidencia estadística que demuestre que haya diferencias significativas para las fases lunares ( $\mathrm{p}=0.950>0.05$ ), siendo en luna menguante más favorable el prendimiento con el $95.833 \%$.

No existe evidencia significativa para la interacción fases lunares* técnicas de injertos, $(\mathrm{p}=0.761>0.05)$, siendo en luna creciente factible para obtener el mayor prendimiento en la técnica tipo yema (100\%), mientras que la luna menguante propicia el mejor prendimiento en el injerto tipo bisel (93.057\%).

\section{Recomendaciones}

En relación al prendimiento de las dos técnicas de injertación en las diferentes fases lunares se recomienda utilizar la técnica tipo yema en la fase lunar creciente, ya que se obtienen los mayores prendimientos (100\%).

En cuanto a los efectos de las fases lunares en los dos tipos de injertos, las medias apareadas de ambas técnicas de injertación indican que la luna menguante propicia los mayores prendimientos con el $95.83 \%$. 
Para próximos ensayos considerar otras variables como vigorización, también incluir en el estudio la evaluación de más factores como clones.

Es necesario validar estos resultados, bajo otras condiciones edafoclimaticas, material genético, época de injertación, para tener información más completa.

\section{Lista de referencias}

Angles, J. (1996). Influencia de la luna en la agricultura. (5 ed.). Mundi-Prensa.

Borbotó, V. A. (2014). Influencia de las fases lunares sobre la reproducción vegetativa de ramillas de diferentes variedades de cacao (Theobroma cacao L.) en la zona de Babahoyo. Babahoyo, Ecuador.

Calderón, L. M. (2012). Influencia de las fases lunares en el crecimiento y rendimiento de cuatro variedades de arveja (Pisum sativum L.) sembradas a doble excavado y de forma tradicional, en San Ignacio, canton Antonio Ante. Ibarra, Ecuador.

Cantero, J. I. (2012). Comportamiento ecofisiológico de cuatro clones de cacao (Theobroma cacao) propagados mediante tres métodos de injertación en el CURDN en ArmeroGuayabal,Tolima. Armero Guayabal, Tolima, Colombia.

CORPOICA. (2004). Propagación asexual de plantas. Bogotá, Colombia.

Corral, J. A. (2012). Influencia del portainjeros en la calidad del pimiento "tipo ramiro" en invernadero. Almeria, España.

Durán, C. R. (2009). Efecto de tres sistemas de injertación y cuatro fases lunares en la obtención de plantas injertadas de durazno (prunus persica) en Ibarra,provincia de Imbabura. El Angel, Ecuador.

Márquez, A. A. (2001). Crecimiento de la papaya (carica papaya) en las diferentes fases de la luna en la zona atlántica de Costa Rica. Guácimo, Costa Rica.

Molina, T. I. (2008). Estudio de diferentes sistemas de injerto examinado al efecto de las fases lunares sobre la multiplicación asexual de cacao (Theobroma cacao L.). Babahoyo, Ecuador.

Montes, A. F. (2010). Manual agroclimático,para la realización de injertos en árboles frutales caducifolios de clima frio-templado,para principiantes. México. 
Muñoz, V. D. (2013). Evaluación del prendimiento del injerto de naranjilla (Solanum quitoense) en dos porta injertos (Solanum arboreum, solanum hirtum) en las cuatro fases lunares en la zona agroecológica de caluma. Guaranda, Ecuador.

Muse, J. A. (2012). Evaluación de métodos de injertación para generar nuevo material productivo para la vid de mesa. Santiago, chile.

Olivo, D. W. (2013). Evaluación del desarrollo de estacas de Mora de Castilla (Rubusglaucus b) con tres tipos de sustratos en las cuatro fases lunares en el cantón Chillanes. Guaranda, Ecuador.

Orantes, K. M. (2003). Desarrollo de portainjerto y evaluación del prendimiento de injerto en anona comun (Anona diversifolia) utilizando diferentes fertilizantes foliares y al suelo. San Salvador, El Salvador.

Palma, M. M. (2009). Evaluación de métodos de injertación en genotipos de tomate (Lycopersicon spp). Xoxocotlán, Oaxaca, México.

Paredes, R. O. (2010). Propagación vegetativa por injerto de Bolaina blanca (Guazuma crinita Mart) bajo condiciones controladas en pucallpa, Perú. Tingo Maria, Perú.

Restrepo, J. (2005). La luna y su influencia en la agricultura.

Rojas, Oscar Paredes (2010). Propagación vegetativa por injerto de bolaina blanca (Guazuma crinita mart.) Bajo condiciones controladas en Pucallpa, Perú. Disponible en: http:// www.iiap.org.pe/cdpublicaciones2011/documentos/pdf/probosques/pu/73.pdf

Santana, W. M. (2013). Determinación del tipo de injerto y la hora efectiva de realización,sobre el prendimiento de yemas en plántulas de cacao en la zona de pueblo viejo, provincia de los rios. Babahoyo, Ecuador.

Sian, J. L. (2005). Evaluación del prendimiento de injerto de cacao (Theobroma cacao) Uf667, en cinco etapas de crecimiento del patrón Pound - 7. San carlos, Guatemala. 